PROCEEDINGS OF THE

AMERICAN MATHEMATICAL SOCIETY

Volume 135, Number 1, January 2007, Pages 163-168

S 0002-9939(06)08446-2

Article electronically published on June 20, 2006

\title{
ON SUBMULTIPLICATIVITY OF SPECTRAL RADIUS AND TRANSITIVITY OF SEMIGROUPS
}

\author{
HEYDAR RADJAVI AND PETER ROSENTHAL
}

(Communicated by Joseph A. Ball)

\begin{abstract}
It is shown that a transitive, closed, homogeneous semigroup of linear transformations on a finite-dimensional space either has zero divisors or is simultaneously similar to a group consisting of scalar multiples of unitary transformations. The proof begins with the result that for each closed homogeneous semigroup with no zero divisors there is a $k$ such that the spectral radius satisfies $r(A B) \leq k r(A) r(B)$ for all $A$ and $B$ in the semigroup.

It is also shown that the spectral radius is not $k$-submultiplicative on any transitive semigroup of compact operators.
\end{abstract}

\section{INTRODUCTION}

We consider semigroups of linear transformations or operators, by which we simply mean collections closed under multiplication. A semigroup $\mathcal{S}$ is said to be transitive if $\{A x: A \in \mathcal{S}\}$ is dense in the space for every non-zero vector $x$ (this is sometimes called 'topological transitivity', to distinguish it from the case where $\{A x: A \in \mathcal{S}\}$ is the entire space for each $x \neq 0$ ). A semigroup is said to be irreducible if it has no non-trivial invariant subspaces; a transitive semigroup is irreducible (but an irreducible semigroup need not be transitive).

The spectral radius $r$ is said to be $k$-submultiplicative on a semigroup if $r(A B) \leq$ $\operatorname{kr}(A) r(B)$ for all $A$ and $B$ in the semigroup. This paper was partially stimulated by [2, which contains results relating $k$-submultiplicativity of spectral radius to $\frac{1}{k}$-supermultiplicativity (i.e., $r(A B) \geq \frac{1}{k} r(A) r(B)$ ).

We begin with several results on finite-dimensional spaces and then establish a theorem for semigroups of compact operators.

\section{Semigroups of Linear transformations ON FINITE-DIMENSIONAL SPACES}

In this section we restrict our attention to linear operators on finite-dimensional complex vector spaces.

Definition 2.1. A semigroup of linear transformations is said to be homogeneous if it is closed under multiplication by complex scalars. By a closed semigroup we mean one that is closed in the norm topology.

Received by the editors March 22, 2005 and, in revised form, July 27, 2005.

2000 Mathematics Subject Classification. Primary 47D03.

(C)2006 American Mathematical Society Reverts to public domain 28 years from publication 
Theorem 2.2. If a closed, homogeneous semigroup has no (non-zero) zero divisors, then there exists a $k \geq 1$ such that

$$
\frac{1}{k} r(A) r(B) \leq r(A B) \leq k r(A) r(B)
$$

for all $A$ and $B$ in the semigroup.

Proof. Suppose there is no $k$ satisfying $r(A B) \leq k r(A) r(B)$ for all $A$ and $B$. For each $n$, choose a pair $\left\{A_{n}, B_{n}\right\}$ in the semigroup such that

$$
r\left(A_{n} B_{n}\right)>n r\left(A_{n}\right) r\left(B_{n}\right) .
$$

Dividing by $r\left(A_{n}\right) r\left(B_{n}\right)$ yields operators $C_{n}$ and $D_{n}$ with $r\left(C_{n}\right)=r\left(D_{n}\right)=1$ and

$$
r\left(C_{n} D_{n}\right)>n .
$$

Thus, at least one of the sequences $\left\{\left\|C_{n}\right\|\right\}$ and $\left\{\left\|D_{n}\right\|\right\}$ is unbounded. Assume, after replacing sequences by subsequences, that

$$
\left\|C_{n}\right\| \rightarrow \infty \text { and } \frac{C_{n}}{\left\|C_{n}\right\|} \rightarrow R \text {. }
$$

Now, $R \neq 0$ (because $\|R\|=1$ ) and, by continuity of the spectral radius, $r(R)=$ 0 . Hence $R$ is nilpotent, so the semigroup has zero divisors, which is a contradiction. Thus there is a $k$ such that

$$
r(A B) \leq k r(A) r(B)
$$

for all $A$ and $B$ in the semigroup. Since the semigroup contains members $A$ with spectral radius one, and since $r\left(A^{2}\right)=r(A)^{2}$, it follows that $k \geq 1$. The fact that

$$
r(A B) \geq \frac{1}{k} r(A) r(B)
$$

then follows from Theorem 2 of 2 .

Theorem 2.3. A transitive, homogeneous, closed semigroup that has no zero divisors is simultaneously similar to a group consisting of scalar multiples of unitary transformations.

Proof. Let $\mathcal{S}$ be such a semigroup.

By Lemma 3.1.6 of [3], since the semigroup is irreducible, there is an idempotent $E$ of minimal non-zero rank in $\mathcal{S}$ such that the restriction of $E \mathcal{S} E \backslash\{0\}$ to the range of $E$ is simultaneously similar to a group of multiples of unitaries. If $E$ is the identity operator, there is nothing more to prove. Suppose otherwise; it must then be shown that $\mathcal{S}$ contains zero divisors.

Perform a similarity so that the restriction of $E \mathcal{S} E$ to the range of $E$ consists of multiples of unitaries. Decompose members of $\mathcal{S}$ as block matrices with respect to the ranges of $E$ and $I-E$; each $(1,1)$ entry in this block decomposition is a multiple of a unitary operator.

Suppose there are no zero divisors in $\mathcal{S}$. By Theorem 2.2 above, there is then a $k$ such that

$$
r(A B) \leq k r(A) r(B)
$$

for all $A$ and $B$ in $\mathcal{S}$. We will show that the transitivity of $\mathcal{S}$ implies there is no such $k$.

Since $\mathcal{S}$ is transitive, there is a $T$ other than 0 in $\mathcal{S}$ whose $(1,2)$ block entry $T_{12}$ is not zero. Then $T$ 's $(1,1)$ block $T_{11}$ is also different from zero, since otherwise $E T$ 
would be a non-trivial nilpotent member of $\mathcal{S}$. Multiply $T$ by an appropriate scalar so that $T_{11}$ is unitary, and call the resulting operator $R$. The operator $E R$ has the block form

$$
\left(\begin{array}{cc}
R_{11} & R_{12} \\
0 & 0
\end{array}\right)
$$

with $R_{11}$ unitary and $R_{12} \neq 0$. Choose a vector $g$ in the range of $I-E$ such that $R_{12} g \neq 0$.

Let $s$ and $t$ be positive numbers, to be specified later. Fix any unit vector $f$ in the range of $E$. By the transitivity of $\mathcal{S}$, we can find $S$ in $\mathcal{S}$ so that $S f$ is within $t$ of $s g$. Then

$$
\left\|S_{11} f\right\|<t \text { and }\left\|S_{21} f-s g\right\|<t .
$$

Since $f$ is a unit vector and $S_{11}$ is a multiple of a unitary operator, it follows that $\left\|S_{11}\right\|<t$.

Let $A=S E$ and $B=E R$. Then $r(A)=r\left(S_{11}\right)<t$ and $r(B)=r\left(U_{11}\right)=1$. Also,

$$
B A=E R S E=\left(\begin{array}{cc}
R_{11} S_{11}+R_{12} S_{21} & 0 \\
0 & 0
\end{array}\right)
$$

Hence,

$$
B A f=\left(R_{11} S_{11}+R_{12} S_{21}\right) f .
$$

By choosing $t$ sufficiently small, $B A f$ can be made as close to $s R_{12} g$ as desired (by the above inequalities). Thus the norm of $B A$ may be made arbitrarily large by choosing $s$ sufficiently large.

This leads to a contradiction, as follows: By the above, $r(A)<t$ and $r(B)=1$. Note that $r(B A)=\|B A\|$, since the $(1,1)$ block of $B A$ is a multiple of a unitary operator and the other entries are 0 . Thus choosing $s$ sufficiently large yields

$$
r(B A)>k r(B) r(A) .
$$

Theorem 2.4. If $\mathcal{S}$ is a transitive semigroup of linear transformations on which the spectral radius is $k$-submultiplicative for some $k$, then there is an invertible transformation $A$ such that $\left\{A^{-1} S A: S \in \mathcal{S}\right\}$ consists of multiples of unitary transformations.

Proof. Let $\mathcal{J}$ be the closure of $\mathbb{C S}$. Note that continuity of the spectral radius implies that $\mathcal{J}$ also satisfies the hypothesis. Assume that the spectral radius is not multiplicative. Then Theorem 2.3 together with this assumption implies that there are non-zero $A$ and $B$ in $\mathcal{S}$ with $A B=0$. Thus the set $B \mathcal{S} A$ consists of operators $X$ with $X^{2}=0$. Since $B \mathcal{S} A \neq\{0\}$ (by the irreducibility of $\mathcal{S}$ ), the set $\mathcal{N}$ of nilpotent members of $\mathcal{S}$ is different from $\{0\}$. Now the $k$-submultiplicativity of spectral radius on $\mathcal{S}$ implies that $\mathcal{N}$ is a semigroup ideal of $\mathcal{S}$. Since $\mathcal{N}$ is triangularizable by Levitzki's Theorem ([1], Theorem 2.1.7 of [3]), and since every non-trivial ideal of an irreducible semigroup is irreducible (see Lemma 2.1.10 of [3]), this is the desired contradiction.

Corollary 2.5. If the spectral radius is $k$-submultiplicative on a transitive semigroup of linear transformations, then the spectral radius is multiplicative on the semigroup.

Proof. This follows immediately from Theorem 2.4 
As shown in [2], for a closed homogeneous semigroup $\mathcal{S}$ with no zero divisors and a number $k \geq 1$, the following statements are equivalent:

(1) $r(A B) \leq k r(A) r(B)$ for all $A$ and $B$ in $\mathcal{S}$,

(2) $r(A B) \geq \frac{1}{k} r(A) r(B)$ for all $A$ and $B$ in $\mathcal{S}$.

The following result shows that for every $k$, there is a semigroup 'sharply' satisfying (1) and (2). (An example with $k=4$ is contained in [2].)

Proposition 2.6. Let $m \geq 1$ be given. There is an irreducible semigroup $\mathcal{S}$ with no zero divisors that satisfies (1) and (2) above for $k=m$ but does not satisfy those inequalities for any $k<m$.

Proof. For a non-zero column vector

$$
x=\left(\begin{array}{c}
x_{1} \\
x_{2} \\
\cdot \\
\cdot \\
\cdot \\
x_{n}
\end{array}\right) \text { and a positive number } v
$$

we say that $x$ is of variation $v$ if

$$
\left|\frac{x_{i}}{x_{j}}\right| \leq v
$$

for all $i$ and $j$. (Note that this implies $v \geq 1$ and $x_{i} \neq 0$ for all $i$.)

Let $\chi_{v}$ be the set of all column vectors of variation $v$. It is easily seen that $\chi_{v}$ is a closed set in $\mathbb{C}^{n}$ and $\alpha x \in \chi_{v}$ if $x \in \chi_{v}$ and $0 \neq \alpha \in \mathbb{C}$. Now define $\mathcal{S}_{v}$ to be the set of all matrices with exactly one column from $\chi_{v}$ whose other columns are all zero.

Each member of $\mathcal{S}_{v}$ is of the form $x e_{j}^{*}$, where $x \in \chi_{v}$ and where $\left\{e_{j}\right\}$ are the basis unit columns (with the $j$-th component of $e_{j}$ equal to 1 ). If $x$ and $y$ are members of $\chi_{v}$ and $i, j$ are integers in $[1, n]$, then

$$
\left(x e_{i}^{*}\right)\left(y e_{j}^{*}\right)=\left(e_{i}^{*} y\right) x e_{j}^{*} .
$$

This shows that $\mathcal{S}_{v}$ is a semigroup. Since

$$
r\left(x e_{i}^{*}\right)=\left|x_{i}\right|=\left|e_{i}^{*} x\right|,
$$

where $x_{i}$ is the $i$-th component of $x$, we have

$$
\begin{aligned}
r\left(\left(x e_{i}^{*}\right)\left(y e_{j}^{*}\right)\right) & =\left|e_{i}^{*} y\right| \cdot\left|x_{j}\right|=\left|y_{i}\right| \cdot\left|x_{j}\right| \\
& =\left|\frac{y_{i}}{y_{j}}\right| \cdot\left|\frac{x_{j}}{x_{i}}\right| \cdot\left|x_{i}\right| \cdot\left|y_{j}\right| \\
& \leq v^{2} r\left(x e_{i}^{*}\right) r\left(y e_{j}^{*}\right),
\end{aligned}
$$

which shows that $\mathcal{S}$ satisfies (1) above with $k=v^{2}$. Thus (2) is also satisfied since $\mathcal{S}$ contains no zero divisors. It is easy to see that no $k<v^{2}$ would do: just consider

$$
A=\left(\begin{array}{cccccc}
1 & 0 & 0 & . & . & 0 \\
v & 0 & 0 & . & . & 0 \\
. & . & . & . & . & . \\
. & . & . & . & . & . \\
1 & 0 & 0 & . & . & 0
\end{array}\right) \text { and } B=\left(\begin{array}{cccccc}
0 & v & 0 & . & . & 0 \\
0 & 1 & 0 & . & . & 0 \\
. & . & . & . & . & . \\
. & . & . & . & . & . \\
0 & 1 & 0 & . & . & 0
\end{array}\right) \text {. }
$$

Then $r(A)=r(B)=1$, and $r(A B)=v^{2}$. 


\section{Semigroups of COMpaCt operators}

One of the above theorems can be extended to semigroups of compact operators on Hilbert spaces.

Theorem 3.1. If $\mathcal{S}$ is a transitive semigroup of bounded linear operators on a Hilbert space, and if $\mathcal{S}$ contains a compact operator other than 0 , then the spectral radius is not $k$-submultiplicative on $\mathcal{S}$ for any $k$.

Proof. Suppose there is a $k$ such that

$$
r(A B) \leq k r(A) r(B)
$$

for all $A$ and $B$ in $\mathcal{S}$. Since a non-zero semigroup ideal of a transitive semigroup is transitive, we can and do assume that $\mathcal{S}$ consists of compact operators. Turovski's Theorem (4], 3], Theorem 8.1.11) states that a semigroup of quasinilpotent compact operators has a non-trivial invariant subspace, so $\mathcal{S}$ must contain operators that are not quasinilpotent.

Enlarge $\mathcal{S}$ by including all scalar multiples of the members of $\mathcal{S}$ and then taking the uniform closure; let $\mathcal{J}$ be the enlarged semigroup. Since spectral radius is continuous on the collection of compact operators,

$$
r(A B) \leq k r(A) r(B)
$$

for $A, B \in \mathcal{J}$. By Corollary 8.1.12 of [3], $\mathcal{J}$ contains finite-rank operators other than 0 , and Lemma 8.1.15 of [3] yields a finite-rank idempotent $E$ in $\mathcal{J}$ of minimal non-zero rank.

It follows from the finite-dimensional case that the restriction of $E \mathcal{J} E \backslash\{0\}$ to the range of $E$ is simultaneously similar to a subgroup of multiples of unitary operators.

Now the proof proceeds along the lines of the last part of the proof of Theorem 2.3 above. Since $E$ is of finite rank, and thus is not the identity operator, the transitivity of $\mathcal{J}$ contradicts the $k$-submultiplicativity of the spectral radius.

Corollary 3.2. If $\mathcal{S}$ is a semigroup of bounded linear operators on a Hilbert space that contains a compact operator other than 0 , and if the spectral radius is $k$ submultiplicative on $\mathcal{S}$ for some $k$, then there is a closed subset of the Hilbert space that is invariant under all the operators in $\mathcal{S}$ and is different from $\{0\}$ and the entire space.

Proof. If $\mathcal{S}$ did not have such a proper, closed, invariant set, then $\mathcal{S}$ would be transitive, contradicting the previous theorem.

Proposition 3.3. Let $k \geq 1$. There is an irreducible semigroup of finite-rank operators on $l^{2}$ which has no zero divisors and satisfies

$$
\frac{1}{k} r(A) r(B) \leq r(A B) \leq k r(A) r(B)
$$

for all $A$ and $B$ in the semigroup.

Proof. The example $\mathcal{S}_{v}$ of Proposition 2.6 with $v=\sqrt{k}$ does not occur in infinite dimensions, since $l^{2}$ contains no vectors of any fixed variation. There are known semigroups of finite-rank operators, with no zero divisors on $l^{2}$, with multiplicative spectral radius. Let $\mathcal{J}$ be such a semigroup (e.g. see Example 8.6.6 of [3]). Let $\mathcal{S}_{v}$ 
be the example of Proposition 2.6, with $v=\sqrt{k}$ on a space of dimension 2. Now let $\mathcal{S}=\mathcal{S}_{v} \otimes \mathcal{J}\left(\right.$ on $l^{2} \oplus l^{2}$, identified with $\left.l^{2}\right)$.

The irreducibility of $\mathcal{S}$ and its lack of zero divisors follow from the same properties of $\mathcal{S}_{v}$ and $\mathcal{J}$. All that is left to prove is that if $\mathcal{S}_{i}, i=1,2$ are semigroups satisfying

$$
\frac{1}{k_{i}} r(A) r(B) \leq r(A B) \leq k_{i} r(A) r(B)
$$

for all $A$ and $B$ in $\mathcal{S}_{i}, i=1,2$, then the members of $\mathcal{S}_{1} \otimes \mathcal{S}_{2}$ satisfy

$$
\frac{1}{k_{1} k_{2}} r(A) r(B) \leq r(A B) \leq k_{1} k_{2} r(A) r(B) \text {. }
$$

But since $r(S \otimes T)=r(S) r(T)$ for all $S$ and $T$, we have, for $A_{1}, B_{1}$ in $\mathcal{S}_{1}$ and $A_{2}, B_{2} \in \mathcal{S}_{2}$, that

$$
\begin{aligned}
r\left(\left(A_{1} \otimes A_{2}\right)\left(B_{1} \otimes B_{2}\right)\right) & =r\left(A_{1} B_{1} \otimes A_{2} B_{2}\right)=r\left(A_{1} B_{1}\right) \cdot r\left(A_{2} B_{2}\right) \\
& \leq k_{1} r\left(A_{1}\right) r\left(B_{1}\right) \cdot k_{2} r\left(A_{2}\right) r\left(B_{2}\right) \\
& =k_{1} k_{2} r\left(A_{1}\right) r\left(A_{2}\right) \cdot r\left(B_{1}\right) r\left(B_{2}\right) \\
& =k_{1} k_{2} r\left(A_{1} \otimes A_{2}\right) \cdot r\left(B_{1} \otimes B_{2}\right) .
\end{aligned}
$$

The inequality involving $\frac{1}{k_{1} k_{2}}$ can be similarly established.

\section{REFERENCES}

[1] J. Levitzki, Uber nilpotente Unterringe, Math. Ann. 105 (1931), 620-627. MR1512728

[2] M. Omladic, H. Radjavi, P. Rosenthal and A. Sourour, Inequalities for products of spectral radii, Proc. Amer. Math. Soc. 129 (2001), 2239-2243. MR.1695115 (2001i:47003)

[3] H. Radjavi and P. Rosenthal, Simultaneous Triangularization, Springer-Verlag, New York, 2000. MR1736065 (2001e:47001)

[4] Y.V. Turovski, Volterra semigroups have invariant subspaces, J. Funct. Anal. 162 (1999), 313-322. MR1682061(2000d:47017)

Department of Pure Mathematics, University of Waterloo, Waterloo, Ontario, CANADA N2L 3G1

E-mail address: hradjavi@cpu105.math.uwaterloo.ca

Department of Mathematics, University of Toronto, Toronto, Ontario, Canada M5S $3 \mathrm{G} 3$

E-mail address: rosent@math.toronto.edu 